Document downloaded from:

http://hdl.handle.net/10251/67208

This paper must be cited as:

Pérez Pérez, MF.; Valderas Aranda, PJ.; Fons Cors, JJ. (2013). A Domain Specific Language for Enabling Doctors to Specify Biomechanical Protocols. IEEE Symposium on Visual Languages and Human-Centric Computing (VL/HCC 2013). IEEE. doi:10.1109/VLHCC.2013.6645251.

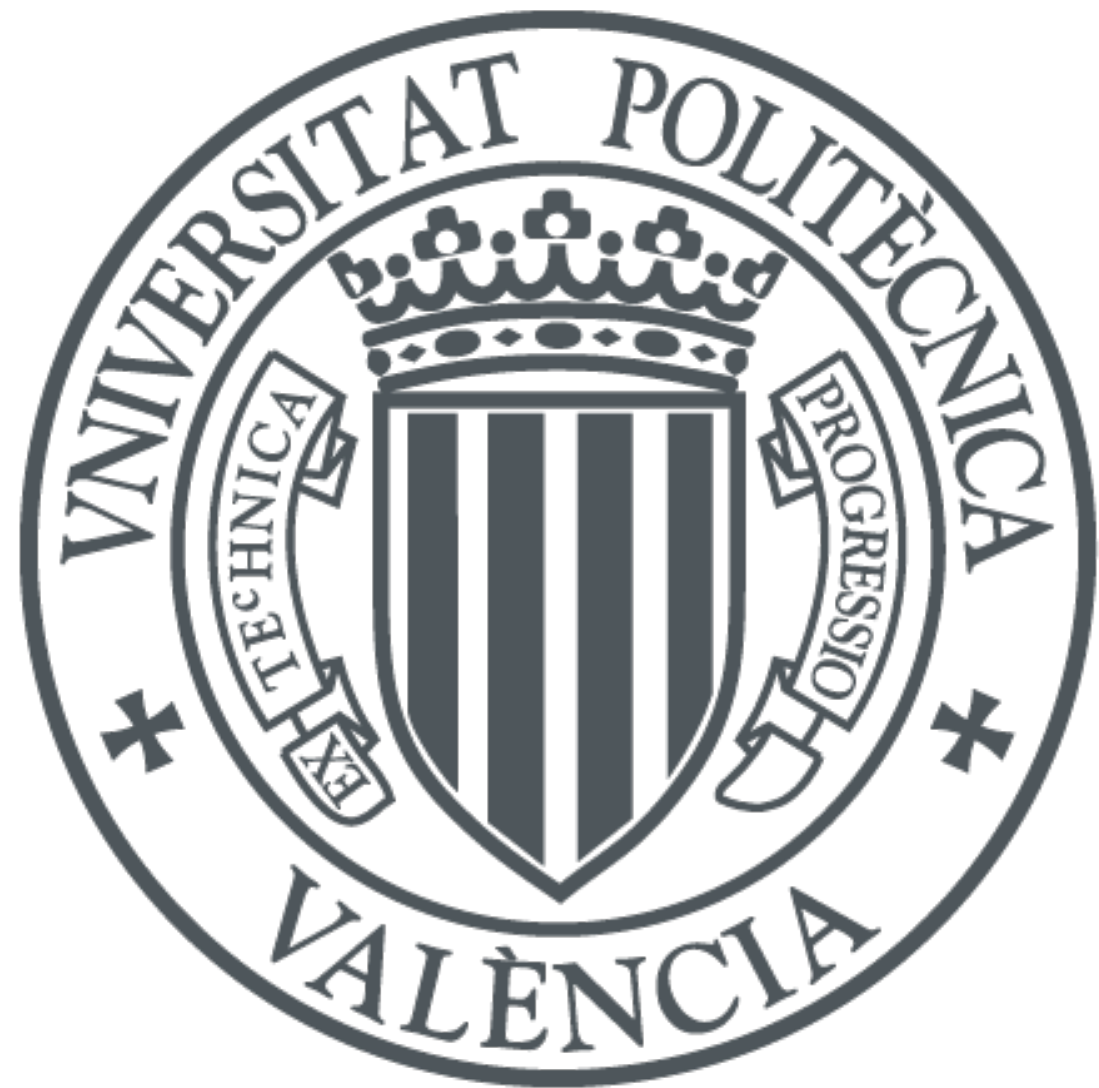

The final publication is available at

http://ieeexplore.ieee.org/xpl/mostRecentlssue.jsp?punumber=6631549

\title{
Copyright IEEE
}

Additional Information

"C2013 IEEE. Personal use of this material is permitted. Permission from IEEE must be obtained for all other uses, in any current or future media, including reprinting/republishing this material for advertising or promotional purposes, creating new collective works, for resale or redistribution to servers or lists, or reuse of any copyrighted component of this work in other works. 


\title{
A Domain-Specific Language for Enabling Doctors to Specify Biomechanical Protocols
}

\author{
Francisca Pérez ${ }^{* \dagger}$, Pedro Valderas ${ }^{\dagger}$, Joan Fons ${ }^{\dagger}$ \\ *Escuela Politécnica Superior \\ Universidad San Jorge \\ Autovía A-23 Zaragoza-Huesca Km.299, 50830 Villanueva de Gállego (Zaragoza), Spain \\ Email: mfperez@usj.es \\ ${ }^{\dagger}$ Centro de Investigación en Métodos de Producción de Software \\ Universitat Politècnica de València \\ Camino de Vera, s/n, 46022 Valencia, Spain \\ Email: pvalderas@pros.upv.es, jjfons@pros.upv.es
}

\begin{abstract}
New technologies are entering medical practice at an astounding pace. However, these technologies often cause to doctors learn and use difficulties. Then, doctors require assistance of a biomedical engineer. This is currently happening in a local hospital that has new technology to analyze biomechanical protocols in patients. Protocols are used to measure performances and identify changes in human body movements and muscles. Doctors are neither familiar with the concepts nor tools used, so biomedical engineers carry out descriptions of protocols rather than doctors. In this paper, we present the design of a domainspecific language that enables doctors to specify biomechanical protocols by addressing learning barriers (using design patterns). We also make doctors' descriptions compatible with the existing tools, and we also support legacy biomedical descriptions (combining meta-modeling and model transformations).
\end{abstract}

\section{INTRODUCTION}

New technologies are emerging constantly in many fields such as health. More and more hospitals are using new technologies that are often advantageous for the patient but health professionals may depend on the knowledge and expertise of biomedical engineers to carry out their tasks [1].

To illustrate this dependence on using new technology between health professionals and biomedical engineers, we present a current scenario in a local hospital. Doctors are provided with equipment that is made of tools, cameras, and sensors to describe and analyze biomechanical protocols. Biomechanical protocols are used to measure performances and identify changes in human body movements and muscles. For example, doctors can detect movements that produce pain or not in patients. Although these tools provide advantages for the patient, doctors do not use them due to the complexity, so they depend on biomedical engineers. This is because the tools use concepts that doctors are not familiar with (such as projections, vectors, or signals) while doctors use different concepts according to their medical activity (such as series, movements, or muscles). As a result, biomedical engineers carry out the descriptions of biomechanical protocols according to the doctors' descriptions. This causes a tedious process between doctors and biomedical engineers that could be solved if doctors themselves describe the protocols. To address this, we design a Domain-Specific Language (DSL) that enables doctors to themselves specify biomechanical protocols. To do this, we follow the phases of DSL development [2]: decision, analysis, design, and implementation.

To start with, we decide to design a DSL since, to the best of our knowledge, there is no an existing DSL that fits the concepts of biomechanical protocols. Then, we analyze learning barriers [3] that doctors have (use, design and selection), and we propose a solution to overcome them. Next, we design the DSL (meta-model and view) that uses concepts closer to doctors, and it is inspired by design suggestions and patterns [2], [3], [4], [5], [6]. Finally, we implement an interface prototype. Our goal is not only design a DSL, it is also (1) make doctors' descriptions compatible with the classic tools at the hospital, and (2) provide legacy support for biomedical descriptions. To support this, we combine metamodeling and model transformations.

The remainder of the paper is structured as follows: Section II describes the classic approach at the hospital. Section III overviews the proposed solution. Section IV describes the DSL design. Section V presents the DSL implementation. Finally, Section VI concludes the paper.

\section{BACKGROUND}

The local hospital provides equipment that is made of commercial tools named Capture, Tracker, and Analyzer [7] to describe and analyze biomechanical protocols. It also provides cameras, and sensors to track patients' movements. However, biomedical engineers carry out the descriptions of biomechanical protocols according to the doctors' descriptions. The steps of the classic approach and the relation among patients, doctors, biomedical engineers, and tools are as follows:

1) Doctors describe to biomedical engineers the muscles, movements, or series of movements that they would like to analyze in patients.

2) Biomedical engineers specify biomechanical protocols according to doctors' descriptions in the $A n$ alyzer tool using concepts (such as vectors, projections, signals, and angles rather than concepts used by doctors). The output is a Extensible Markup Language (XML) file that stores the biomechanical protocols. 
3) Patients carry out movements tracked by sensors. For example, knee movements.

4) The Capture tool records patients' movements. In addition, the tool also gets a set of points according to the information of sensors.

5) The Tracker tool gets the correlation (usually as a function of the spatial or temporal distance between the points) and sets names to the stored variables.

6) The Analyzer tool takes as input the XML file and gets a report with the biomechanical protocols analyzed. The report shows graphs, and values used to measure performances and identify changes in human body muscles.

7) Doctors analyze the report. If they detect that some additional information is required or it has to be modified (i.e., add a new movement to analyze), doctors describe to the biomedical engineers again. Then, the process goes back to the Step 1.

The result is a tedious process between doctors and biomedical engineers because there are no doctors' descriptions that have been carried out in an iteration by now. To solve this, doctors should be able to themselves describe biomechanical protocols. This is because doctors are who best know both the protocols they would like to analyze and the information that the report should include to each patient. However, doctors do not use the classic tools, so we analyze why doctors do not use the Analyzer tool and we detect learning barriers using the Ko et al. [3] proposal as follows:

- Design. Doctors would like to analyze a specific knee movement but they do not know how to specify it in the tool. Moreover, the tool does not offer catalogs with predefined movements to help the description of biomechanical protocols.

- Selection. Doctors do not know the concepts to describe body movements using concepts such as track (concept to describe a vector in a plane), vector, model, angle, projections, or signals.

- Use. The tool neither shows information nor descriptions about the required concepts to describe a protocol, so the tool requires a long learning.

Once we have identified the learning barriers in doctors, we are able to propose a solution to overcome them.

\section{SOlution OVERVIEW}

Doctors agree with replacing the existing Analyzer tool with a new interface that enables them to describe biomechanical protocols. To achieve this, we propose that the new interface is supported by a DSL, which overcomes the learning barriers previously identified. In addition, we make doctors' descriptions compatible with classic tools to reuse the protocol analysis and report creation mechanisms. Besides, doctors would like to have legacy support because doctors need to modify and reuse biomedical protocol descriptions that have been described by biomedical engineers using the existing Analyzer tool until now. Therefore, the steps that we propose as solution are as follows (see Fig.1):

1) Doctors themselves describe biomechanical protocols using concepts that they are familiar with.
To do this, doctors use a DSL that is supported by a new interface. Then, doctors' descriptions will be transformed to concepts of the existing Analyzer tool. The output is a Extensible Markup Language (XML) file that stores the description of biomechanical protocols made by doctors.

The remaining Steps 2-5 of the process are as Steps 3-6 of the classic approach because these steps record patients' movements (Step 2), get the points related to the movements (Step 3), set names to the points (Step 4), and get the report (Step 5).

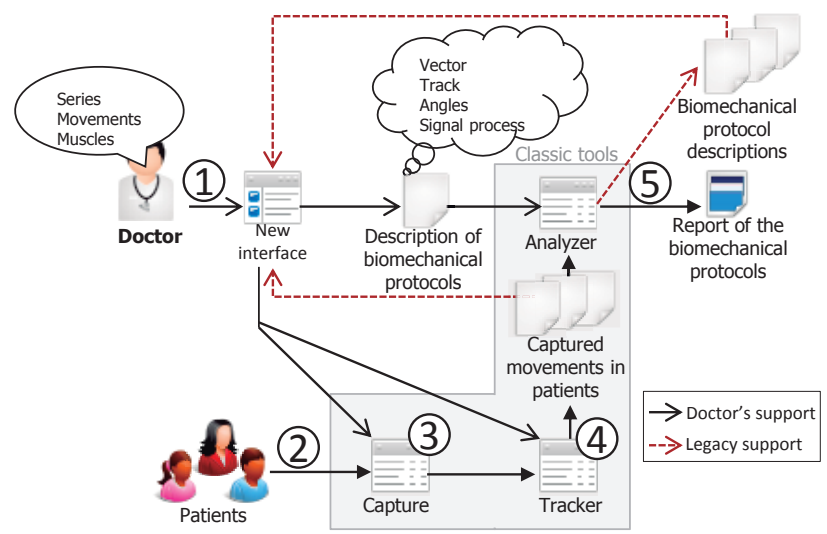

Fig. 1. Solution overview

Note that doctors carry out descriptions of protocols in the new interface using concepts closer to them rather than describe protocols to biomedical engineers. This reduces the steps of the process to 5. In addition, Fig. 1 shows as dotted arrows the legacy support, which enables doctors to modify descriptions that have been previously described by biomedical engineers in the Analyzer tool.

\section{Design of The Domain-Specific LANGUAGE}

As we stated earlier, doctors describe biomechanical protocols using concepts that they are familiar with such as muscles, movements, repetitions, or series (set of movements) rather than using the concepts and the operators that the existing Analyzer tool provides. Fig. 2 shows the main concepts of the DSL. The DSL includes: (1) the concepts that doctors are familiar with (see the upper side of the figure), (2) the concepts that support movement and muscle measures (see the dashed blue boxes in the middle side of the figure), and (3) the concepts that allow doctors to design reports according to their preferences (see the dotted red boxes in the bottom side of the figure). Fig. 2 also shows how the DSL concepts are related. A brief description of the main concepts is as follows:

- Protocol. It is the root concept to measure performances in series of human body movements. It has to be mainly related to a Patient.

- Series. It is composed by one or more movements, which can be repeated one or more times.

- Movement. It represents a movement that can be performed by patients. At the moment, doctors are focused on analyzing knee and shoulder movements, 
so we specialize Movement in Knee and Shoulder. Moreover, we specialize each one with the available existing movements. Shoulder is mainly specialized in Flexion, Extension, Abduction, and Adduction. Knee is mainly specialized in Flexion and Extension. Each movement can be repeated one or more times, and it involves one or more muscles.

- Muscle. It expresses the muscles that can be analyzed in a movement.

- Measure. It represents measures that can be obtained by analyzing Muscle or Movement data.

- Movement Measure. It is related to the movement that analyzes. Moreover, we analyze the data that is usually measured in a movement and we specialize Movement Measure to represent the available measures such as angle, velocity and frequency.

- Muscle Measure. It is related to the muscle that analyzes. Moreover, we also analyze the data that is usually measured in a muscle and we specialize Muscle Measure to represent the available measures such as frequency and RMS (Root Mean Square used to analyze the fatigue).

- Report. It expresses the report that doctors should design to review the output data for the selected measures using plots and tables.

- Plot. It represents data about a measure of a muscle or movement.

- Table. It represents data about measure of a muscle or movement. A table is composed by one or several rows (each row is represented with the RowData concept).

Thus, these concepts provide expressiveness to doctors using concepts they are familiar with rather than force them to learn how to specify each movement and measure using the concepts of the existing Analyzer tool.

\section{A. The DSL View}

We design the DSL view by taking two main design decisions: (1) a catalog of movements and measures, and (2) a wizard. The description of each one and how they are shown is as follows:

1) The catalog of movements and measures: we decide to design a catalog of movements and measures to overcome the design barrier by following the design suggestion proposed by Ko et al. [3]. In addition, we detect that a catalog perfectly fits in the description of biomechanical protocols because we notice that doctors use (1) the same kind of movements (doctors do not create body movements, they use the existing ones), (2) the same muscles are involved in a specific movement, and (3) the same measures can be analyzed in different protocols.

The view of the catalog is different for Movements and Measures. Each Movement is shown as a box with a representative image, name, and the property to set the number of repetitions (the number of repetitions by default is one). Each Measure is displayed using a tree as [4] recommends in a different view to design the report. The tree has two main

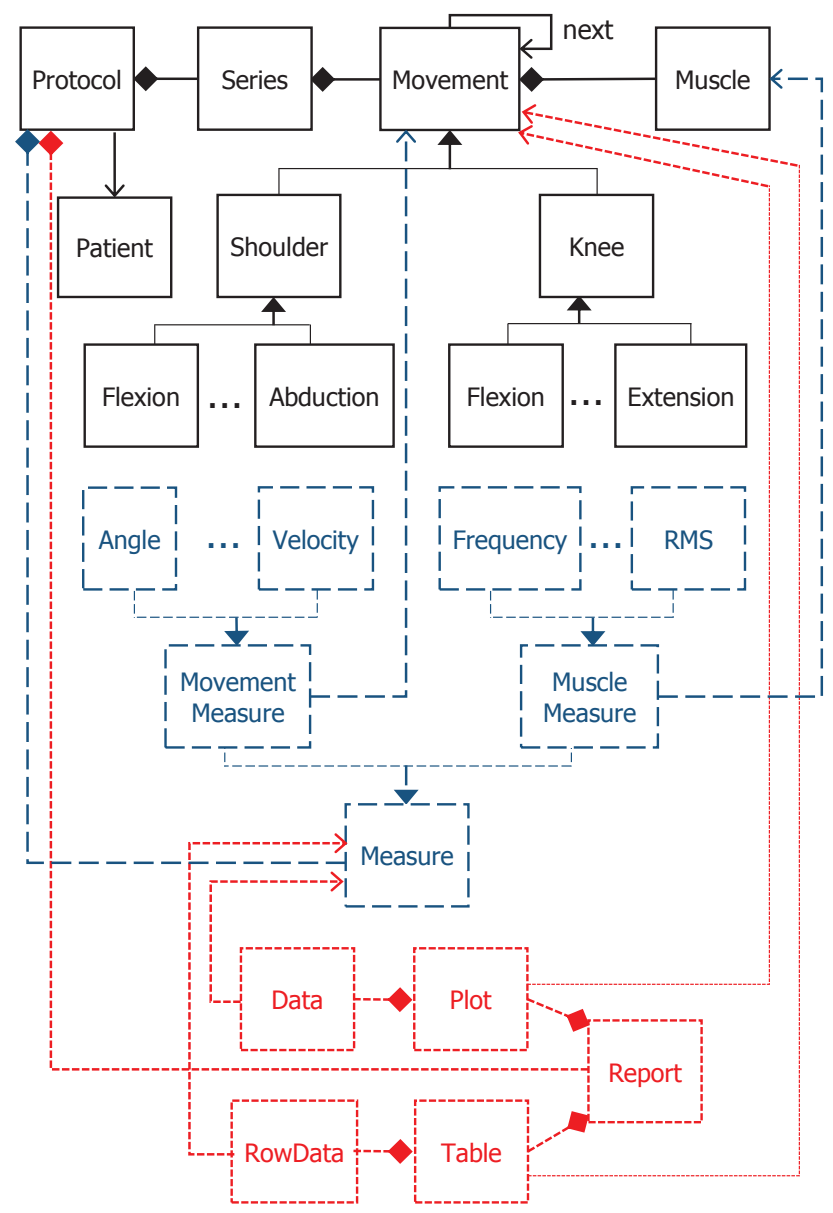

Fig. 2. The DSL meta-model

nodes: Movements and Muscles, which are used to represent the available measures. Each item of the tree includes the measure name and a brief description.

2) The wizard: We decide to include a wizard to overcome the selection and use barriers by following the Welie et al. [4] recommendation. The wizard helps doctors to describe a protocol since several decisions need to be made. In the wizard, we design five steps that doctors should follow to describe a protocol. In addition, we use navigation buttons to guide doctors among the steps. The steps of the wizard are the following:

1) The protocol creation. It allows doctors to create a new protocol description by setting a name to identify it and selecting the body part to analyze (shoulder or knee). For example, a doctor creates a new protocol named Right shoulder protocol and selects shoulder as body part.

2) The series description. It allows doctors to select the movements that describes a series of movements. The available movements are shown from the catalog according to the body part selected in the previous step. For example, the series is specified to be repeated once and it is made up of four movements (flexion, extension, abduction, and adduction). Each movement is specified to be repeated four times.

3) The report definition. It allows doctors to design 
the elements (plots and tables) that the report should include. For example, the report of this protocol includes a table. For each row, an angle movement measure is selected for each movement.

4) The patient selection. It allows doctors to relate the protocol that has just been described for a patient. Thus, the patient can perform the movements according to doctors' descriptions.

5) The protocol storage. It allows doctors to store the protocol. The view of this is just a button. However, we would like to highlight the importance of this step because of it depends that the protocol is (1) stored using the DSL concepts (this enables doctors to reuse protocol descriptions), and (2) transformed into concepts of the existing Analyzer tool (this enables doctors to get a report) according to their descriptions. To do this, we combine meta-modeling and model transformations to design a mapping model that sets correspondences among concepts of the existing tool and concepts of the DSL. Moreover, we design a set of transformation rules that use as input the protocol description and the mapping model to produce as output a XML file compatible with the existing Analyzer tool. The description of the mapping model and the transformation rules is beyond the scope of this paper.

\section{INTERFACE PROTOTYPE}

We implement an interface prototype according to the DSL view presented in the previous section. Fig. 3 shows a snapshot of the interface prototype of The Series Description step. Specifically, the figure shows (1) the head and navigation buttons (see the upper side of the figure); (2) the catalog of movements for shoulder (see the middle side of the figure); (3) the area to describe and set the series of movements and repetitions by using drag and drop from the catalog, and selection for the number of repetitions for each movement (as figure shows, the series of movements is made up of four movements and each one has set 4 repetitions); (4) the doctor's description compatibility with the classic tools once the Step 5 (The Protocol Storage step) of the wizard is done; and (5) the legacy support by means of model transformations.

Finally, it is important to stress that the interface requires that 14 elements have been selected and 26 elements have been set among movements and report elements in total. The existing Analyzer tool requires 93 operators and 128 variables to describe the same protocol. In the Protocol Storage step, doctor's descriptions are embedded in model transformations to obtain the XML file that includes the required operators and variables, which are compatible with the Analyzer tool.

\section{CONCLUSIONS}

We have presented the analysis, design, and prototype implementation of a DSL for enabling doctors to specify biomechanical protocols. To tackle this, we have studied the classic approach at the hospital, and we have identified the learning barriers (design, selection and use) that doctors had using the classic approach to overcome them. The interface and the model transformations provide the first evidences that the DSL is complete, correct and unambiguous.

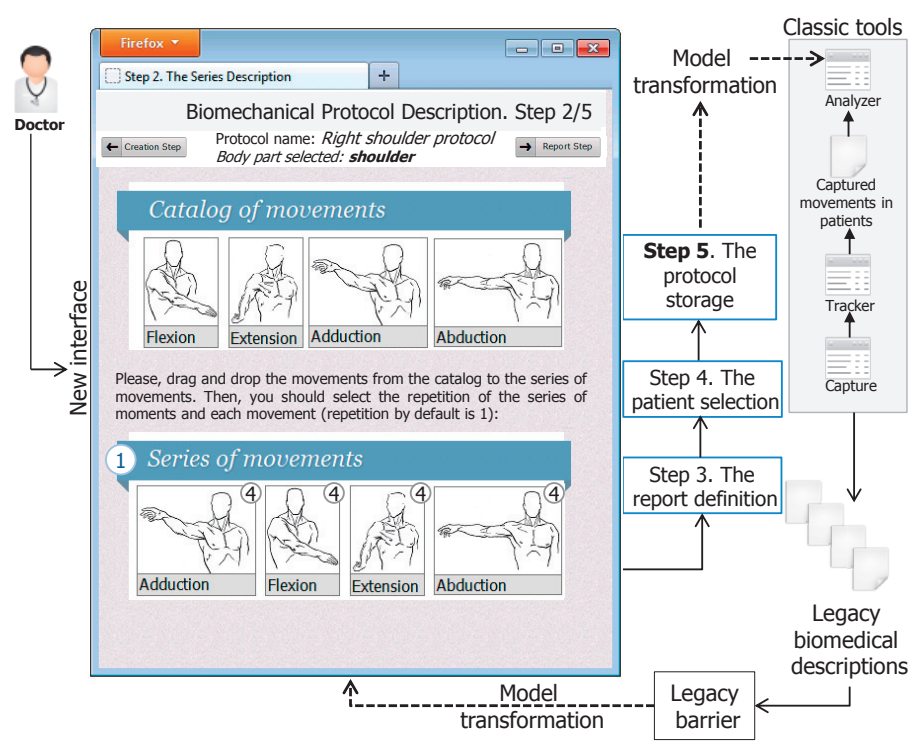

Fig. 3. A snapshot of the prototype interface and process

In short, the main advantages that our DSL provides are: (1) doctors are able to themselves carry out descriptions of biomechanical protocols, (2) doctors manage less concepts, (3) movements and measures are reused rather than force doctors to describe them from scratch, (4) doctors' descriptions are compatible with the classic approach, and (5) existing protocol descriptions can be reused thanks to the legacy support.

\section{ACKNOWLEDGMENTS}

This work has been developed with the support of Ministerio de Ciencia e Innovación (MICINN) under the project EVERYWARE TIN2010-18011 and co-financed with European Regional Development Fund (ERDF). The authors would also like to thank Jesús Carro who provided valuable knowledge about the classic approach and the existing tools at the hospital, and Dr. Andrés Alcazar and his biomedical team who participated and provided feedback in the project.

\section{REFERENCES}

[1] W. H. Organization, "Increasing complexity of medical technology and consequences for training and outcome of care," Geneva : World Health Organization, August 2010.

[2] M. Mernik, J. Heering, and A. M. Sloane, "When and how to develop domain-specific languages," ACM Comput. Surv., vol. 37, no. 4, pp. 316344, Dec. 2005

[3] A. J. Ko, B. A. Myers, and H. H. Aung, "Six learning barriers in end-user programming systems," in Proceedings of the 2004 IEEE Symposium on Visual Languages - Human Centric Computing, ser. VLHCC '04. Washington, DC, USA: IEEE Computer Society, 2004, pp. 199-206. [Online]. Available: http://dx.doi.org/10.1109/VLHCC.2004.47

[4] M. van Welie and H. Trtteberg, "Interaction patterns in user interfaces," in PLoP 2000, 2000, pp. 13-16.

[5] M. P. Couper, R. Tourangeau, F. G. Conrad, and S. D. Crawford, "What they see is what we get: response options for web surveys," Soc. Sci. Comput. Rev., vol. 22, no. 1, pp. 111-127, 2004.

[6] W. O. Galitz, The Essential Guide to User Interface Design: An Introduction to GUI Design Principles and Techniques. New York, NY, USA: John Wiley \& Sons, Inc., 2002.

[7] (2013) Bts bioengineering kinematics. [Online]. Available: http://www.btsbioengineering.com/BTSBioengineering/Kinematics/ BTSSMARTANALYZER/BTS_SMART_ANALYZER.html 